\title{
Is the profile of chronic pancreatitis in India changing?
}

\author{
Saroj K Sinha $\cdot$ Rakesh Kochhar
}

Received: 27 March 2014 / Accepted: 27 March 2014 / Published online: 6 April 2014

(C) Indian Society of Gastroenterology 2014

Chronic pancreatitis (CP) is characterized by irreversible damage to the pancreas that eventually leads to pain and/or exocrine and endocrine insufficiencies [1]. It is usually characterized by a clinical course leading to progressive loss of pancreatic parenchyma, endocrine and exocrine insufficiencies, or formation of stones/calcification in the pancreas [2]. It is a significant health problem worldwide and is associated with considerable morbidity. Prevalence of $\mathrm{CP}$ varies widely in different geographic locations. Compared with 10-15/10 prevalence in Western countries, the prevalence of $\mathrm{CP}$ is higher in India and some of the other Asian countries [3, 4]. An earlier study [4] from India showed the prevalence of CP to be $125 / 10^{5}$. A Chinese study [5] showed increasing prevalence of CP from $3.08 / 10^{5}$ in 1996 to $13.52 / 10^{5}$ population in 2003, and a nationwide survey in Japan [6] showed the prevalence to be $45 / 10^{5}$. The etiology of CP also varies in different countries. Whereas alcohol is the commonest etiology in most of the Western countries, idiopathic chronic pancreatitis (ICP) is the most common type reported from India and China [4]. In earlier reports from India, tropical chronic pancreatitis (TCP) dominated the description of CP [3, 4]. The reported common features of TCP include its onset in childhood and adolescence, prominent association with malnutrition, higher prevalence in population-eating cassava, high prevalence of diabetes mellitus, marked dilatation of pancreatic duct, and formation of pancreatic calculi which are usually large [3, 4]. The disease was reported to have an aggressive course with patients dying in the prime of their lives because of complications related to $\mathrm{CP}$ including pancreatic carcinoma [7-10]. However, the spectrum of CP in India seems to be changing now. As opposed to previous reports

S. K. Sinha $\cdot$ R. Kochhar $(\bowtie)$

Department of Gastroenterology, Postgraduate Institute of Medical

Education and Research, Chandigarh 160 012, India

e-mail: dr_kochhar@hotmail.com of marked ductal dilatation and large calculi, less advanced forms of $\mathrm{CP}$ are being diagnosed now; this may be related to the availability of better diagnostic modalities like CT scan and MRCP. It may also be related to changes in the spectrum of the disease. In fact, only about $3.8 \%$ to $5.8 \%$ of patients could satisfy the criteria of the so-called TCP in recent studies [8-11].

In the current issue of this Journal, Rajesh et al. [12] present their data on CP from Kerala, a state which has been reported earlier to have high prevalence of TCP. The study included 597 cases of CP, of which, 175 were alcoholic chronic pancreatitis (ACP) and 390 were ICP. Some of the patients were included retrospectively. Two hundred five of the 390 patients with ICP were prospectively followed for about 4 years. Among the patients with ICP, $54.1 \%$ had early onset disease and $45.9 \%$ had late-onset disease (cutoff age for the definition being, 30 years). Patients with early onset ICP had symptomatic period of almost 14 years before presentation, but lower frequency of diabetes which developed almost 13 years after onset of symptoms whereas those with lateonset ICP had symptomatic period less than 5 years but higher frequency of diabetes which occurred almost simultaneously with presentation. The early and late-onset disease did not differ with respect to the risk of pancreatic carcinoma or development of pseudocyst. Thus, the two groups of patients seem to have different profiles of the disease. Earlier, Layer et al. [13] also reported that in early onset idiopathic pancreatitis, calcification and exocrine and endocrine insufficiencies developed more slowly than in late-onset idiopathic and alcoholic pancreatitis. It is tempting to think of early onset ICP to be similar to TCP in the current study originating from Kerala, but the current profile of early onset ICP seems different from that reported for TCP. Some of the earlier studies have used 35 years of age for defining early vs. late-onset ICP [14, 15]. In an earlier report from North India which included 155 patients of CP, $41.3 \%$ of patients had ICP and $38.1 \%$ had 
ACP [14]. In the ICP group, $64 \%$ patients had early onset disease (age $<35$ years) and $36 \%$ had late-onset disease (age $>35$ years) which seems comparable to the current study.

The authors also analyzed their data for patients who had early onset disease with respect to alcohol intake. Of the 118 patients with early onset CP, 63 gave history of alcohol intake whereas 55 denied taking alcohol. Non-drinkers of alcohol were younger, had shorter duration of symptoms, and had lower frequency of diabetes mellitus compared to alcohol drinkers. Both groups had similar frequency of calcification. It is intriguing to note that alcohol drinkers had less diabetes mellitus despite a seemingly rapidly progressive disease. The profiles of these two groups of patients appear quite differently - the early onset ICP (which may be equivalent to previously described TCP) seems to have shorter symptomatic course and development of diabetes in relatively smaller percentage of patients whereas those taking alcohol seem to have longer clinical course but higher frequency of diabetes. It can be argued that diabetes is a reflection of longer duration of disease, but the presence of calcification in equal frequency in the two groups negates this preposition.

Is there a difference in $\mathrm{CP}$ in different parts of India? In an earlier study from northern India involving 225 patients with $\mathrm{CP}, \mathrm{ACP}$ and ICP were nearly equally distributed ( $42 \%$ and $43 \%$, respectively) with pancreas divisum alone (10.7\%), pancreas divisum along with excessive alcohol consumption $(1.8 \%)$, and hyperparathyroidism $(2.2 \%)$ being the other etiologies [15]. In that study, $47 \%$ patients had evidence of pancreatic calcification which is sharply in contrast to the frequency of pancreatic calcification reported in the current study $(95.5 \%$ in early onset and $97.9 \%$ in late-onset disease). We are handicapped by the lack of detailed studies from Western and Eastern India. Data from northern India is variable; data from Chandigarh shows near equal prevalence of ACP and ICP, but data from New Delhi shows predominance of ICP $[4,8,14,15]$. This is in contrast to studies from Western countries, Japan, and Australia where ACP is clearly the most common type [4]. Earlier, this was attributed to various dietary factors and malnutrition $[16,17]$. Time trends of $\mathrm{CP}$ in India have shown that the age at diagnosis of fibrocalculous pancreatic diabetes and TCP has increased while that of diabetes secondary to ACP has decreased. The body mass index of fibrocalculous pancreatic diabetes and TCP patients has also significantly increased over the years, while that of ACP patients has not [18]. Severe malnutrition may result in pancreatic atrophy and insufficiency, but usually does not lead to $\mathrm{CP}[4,16,17]$. It has been suggested that malnutrition is a result of $\mathrm{CP}$ and not viceversa $[4,16,17]$. In a prospective study of 120 patients with ICP, only $20.6 \%$ were underweight before the onset of disease while $67 \%$ lost weight subsequently, suggesting that malnutrition was an effect and not a cause of CP [16]. It has to be recognized that with economic development and social changes occurring in India, the prevalence of malnutrition is decreasing and per capita alcohol consumption is increasing. Clinical observations from Kerala where the TCP was first described also show a changing profile of $\mathrm{CP}$ with the patients being much older, less malnourished, less often diabetic, and having less advanced disease in the 2000s than in the 1980s $[4,19]$.

Research in the field CP in India is now moving ahead from the story of malnutrition, protein calorie deprivation, and cassava intake to the molecular and genetic level, a phenomenon similar to many other diseases. SPINK1 gene mutations have been reported to be common in Indian patients with TCP $[4,10,20,21]$. In fact, one study found that $42 \%$ of these patients had SPINK1 mutation, $9 \%$ had CFTR gene mutation, and $41 \%$ had CFTR gene polymorphisms [4, 10]. Minor mutations in the CFTR gene were five times more common compared with healthy controls [4]. Mutations in cathepsin B gene have also been shown to be associated with idiopathic CP in India [20]. Chymotrypsinogen C (CTRC) degrades trypsinogen, and its loss-of-function variants have also been in focus of research [4]. One study involving Indian CP patients reported c.180C $>$ T mutation in the CTRC gene [4].

Thus, this study affirms that TCP remains the most common type of CP in South India. The late-onset type has been shown to have higher frequency of both exocrine and endocrine insufficiencies compared to early onset disease. This may be an important finding, signifying a change in the spectrum of the CP in India, as TCP had been reported to have onset typically below 30 years of age and to be associated with high frequency of exocrine and endocrine insufficiencies. Larger multicenter studies may better define the natural history of $\mathrm{CP}$, frequency of various complications, and risk of malignancy. Genetic factors and mutations in various genes also need to be defined more clearly in $\mathrm{CP}$ as a whole group and its various subsets. So we need to travel a long way before the mystery is resolved.

\section{References}

1. Etemad B, Whitcomb DC. Chronic pancreatitis: diagnosis, classification, and new genetic developments. Gastroenterology. 2001;120: 682-707.

2. Steer ML, Waxman I, Freedman S. Chronic pancreatitis. N Engl J Med. 1995;332:1482-90.

3. Tandon RK, Sato N, Garg PK. Chronic pancreatitis: Asia-Pacific consensus report. J Gastroenterol Hepatol. 2002;17:508-18.

4. Garg PK. Chronic pancreatitis in India and Asia. Curr Gastroenterol Rep. 2012;14:118-24.

5. Wang LW, Li ZS, Li SD, Jin ZD, Zou DW, Chen F. Prevalence and clinical features of chronic pancreatitis in China: a retrospective multicenter analysis over 10 years. Pancreas. 2009;38:248-54.

6. Lin Y, Tamakoshi A, Matsuno S, et al. Nationwide epidemiological survey of chronic pancreatitis in Japan. J Gastroenterol. 2000;35: 136-41. 
7. Balakrishnan V, Nair P, Radhakrishnan L, Narayanan VA. Tropical pancreatitis - a distinct entity, or merely a type of chronic pancreatitis? Indian J Gastroenterol. 2006;25:74-81.

8. Garg PK, Tandon RK. Survey on chronic pancreatitis in the AsiaPacific region. J Gastroenterol Hepatol. 2004;19:998-1004.

9. Tandon RK, Garg PK. Tropical pancreatitis. Dig Dis. 2004;22:258-66.

10. Midha S, Khajuria R, Shastri S, Kabra M, Garg PK. Chronic pancreatitis in India: phenotypic characterization and strong genetic susceptibility due to SPINK1 and CFTR gene mutations. Gut. 2010;59:800-7.

11. Balakrishnan V, Unnikrishnan AG, Thomas V, et al. Chronic pancreatitis. A prospective nationwide study of 1,086 subjects from India. JOP. 2008;9:593-600.

12. Rajesh G, Veena AV, Menon S, Balakrishnan V. Clinical profile of early-onset and late-onset idiopathic chronic pancreatitis in South India. Indian J Gastroenterol 2014;33:doi 10.1007/s12664-013-0421-3.

13. Layer P, Yamamoto H, Kalthoff L, et al. The different courses of early-and late-onset idiopathic and alcoholic pancreatitis. Gastroenterology. 1994;107:1481-7.

14. Bhasin DK, Singh G, Rana SS, et al. Clinical profile of idiopathic chronic pancreatitis in north India. Clin Gastroenterol Hepatol. 2009;7:594-9.
15. Bhasin DK, Rana SS, Chandail VS, Singh G, et al. Clinical profile of calcific and noncalcific chronic pancreatitis in North India. J Clin Gastroenterol. 2011;45:546-50.

16. Garg PK. Chronic pancreatitis in India: untying the nutritional knot. Indian J Gastroenterol. 2011;30:63-5.

17. Brooks SE, Golden MH. The exocrine pancreas in kwashiorkor and marasmus - light and electron microscopy. West Indian Med J. 1992;4:56-60.

18. Mohan V, Farooq S, Deepa M. Prevalence of fibrocalculous pancreatic diabetes in Chennai in South India. JOP. 2008;9: 489-92.

19. Midha S, Singh N, Sachdev V, et al. Cause and effect relationship of malnutrition with idiopathic chronic pancreatitis: prospective casecontrol study. J Gastroenterol Hepatol. 2008;23:1378-83.

20. Mahurkar S, Idris MM, Reddy DN, et al. Association of cathepsin B gene polymorphisms with tropical calcific pancreatitis. Gut. 2006;55: 1270-5.

21. Derikx MH, Szmola R, te Morsche RH, Sunderasan S, Chacko A, Drenth JP. Tropical calcific pancreatitis and its association with CTRC and SPINK1 (p.N34S) variants. Eur J Gastroenterol Hepatol. 2009;21:889-94. 